\title{
Neuroscience models: choose your dimension
}

\author{
New types of 2D and 3D brain models capture more physiology.
}

\author{
Vivien Marx
}

T he brain needs models. Open questions about brain development, neuronal communication and the molecular, cellular and circuit-based underpinnings of afflictions such as neurodegenerative disease, psychiatric conditions and brain tumors lead to an acute need in neuroscience for better models of the human brain. Animal studies can link neuronal mechanisms and behavior; human brain research draws on functional magnetic resonance imaging, cognitive tests or postmortem tissue analysis. Assays and models add to such data ${ }^{1}$.

In two-dimensional (2D) approaches, labs might reprogram stem cells into neurons. In three-dimensional (3D) approaches, they might choose spheroids or organoids, which are akin to miniaturized organ systems. Many labs use more than one model system-perhaps a mouse line and a cell culture model, says Tara Spires-Jones, a dementia researcher at the University of Edinburgh. 2D versus 3D need not be an either/or choice. "We do both, they're both very valuable for different things," says Fred 'Rusty' Gage, who directs The Salk Institute for Biological Studies. To gain control and scale, labs are building 2D assays into a variety of $3 \mathrm{D}$ architectures. These draw on increasing bonds between basic cell biology and bioengineering, says Gage, which is making 2D assays more powerful.

To model brain disease or development, researchers need live human brain cells, which are not readily obtained. They can start with patient skin cells, reprogram them into induced pluripotent stem cells (iPSCs) and then differentiate those into various neuron types. Labs might skip the pluripotent stage and convert fibroblasts directly into induced neurons (iNs). Unlike reprogramming and differentiation, which erases the signs of aging in the source cell, these iNs retain the source cell's epigenetic and transcriptomic signatures. That sounds well suited to modeling of age-related diseases, but iNs are not always right: it is hard to generate organoids with them and they are expensive to culture at scale ${ }^{2}$.

\section{Connecting in a dish}

Neuronal connectivity is often studied in $3 \mathrm{D}$ assays, but Gage and colleagues built a 2D way to do so ${ }^{3}$. In the hippocampus, dentate

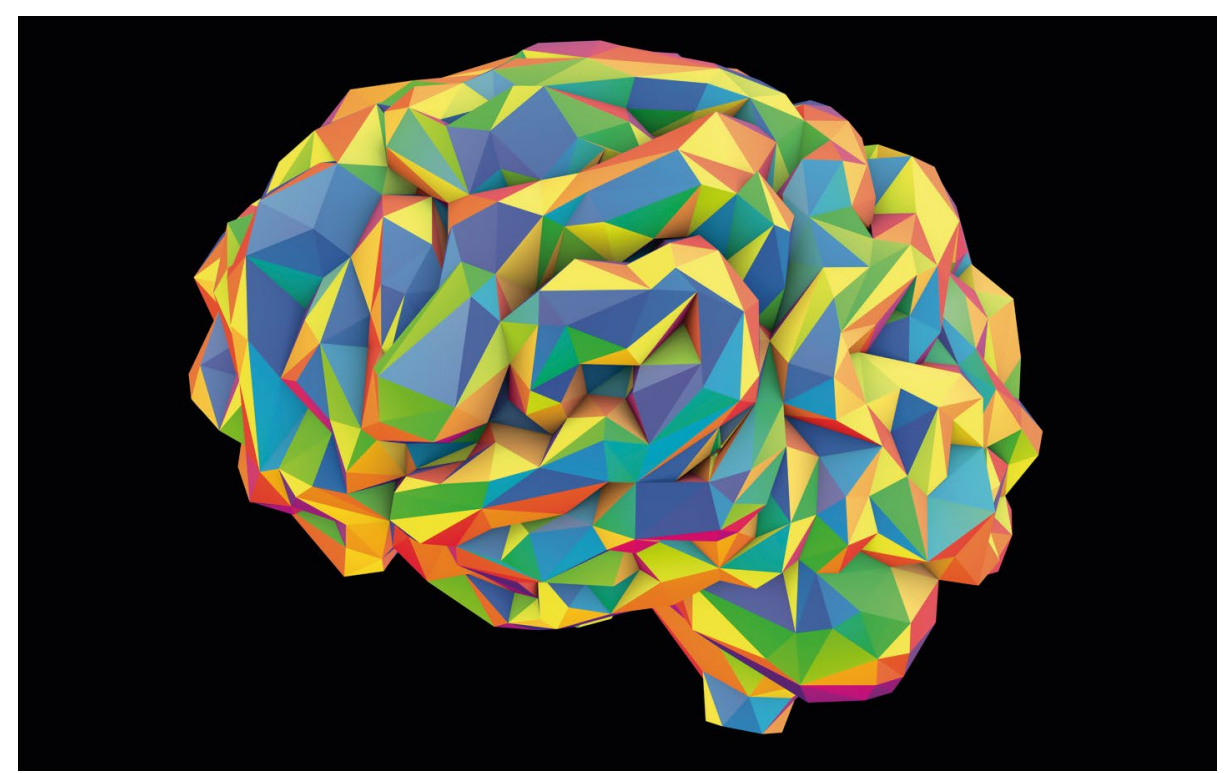

Different types of 2D and 3D assays move scientists toward better models of brain disease or development. Credit: K. Kon/Science Photo Library/Getty

gyrus (DG) neurons send information to CA3 neurons from spatially distinct areas. In the lab's microfluidic assay, DG neurons and CA3 neurons are separated by a barrier with openings through which axons can reach. Using cells from people with schizophrenia, in which altered hippocampal connectivity plays a role, the team generated human iPSCs. They applied the morphogen Wnt3a to differentiate cells into DG and CA3 neurons.

The lab watched how the cell types connect much as the connection occurs in vivo, says Gage. Other researchers, too, have advanced such 2D culture assays, he says, in which labs can study more homogeneous cell populations than in typical 3D setups. "You can control more of the features in a $2 \mathrm{D}$ situation than you can in 3D, where you're sort of left for the biology to recapitulate whatever it does within that context of the sphere or the organoid," he says. Both 2D and 3D recapitulate processes and let labs leverage decades of research on mouse embryonic development such as the emergence of the hippocampus or cortex and the role of transcription factors and morphogens in differentiation and development.

\section{D view}

In Alzheimer's disease (AD), progressive changes in the brain impair cognition and memory. From the postmortem analysis of patient brains, scientists know the hallmarks: atrophied brain tissue, death of neurons and proteinaceous deposits-globs of $\beta$-amyloid peptide and tangles of tau protein fibers. Too little is known about how this state begins and progresses.

Scientists at Massachusetts General Hospital (MGH) and the University of North Carolina (UNC) built a human AD triculture system with neurons, astrocytes and microglia ${ }^{4}$. Neurotoxic inflammation is

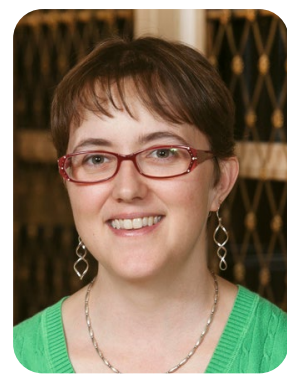

Many labs use more than one model system, says Tara Spires-Jones. (Credit: U. of Edinburgh) 

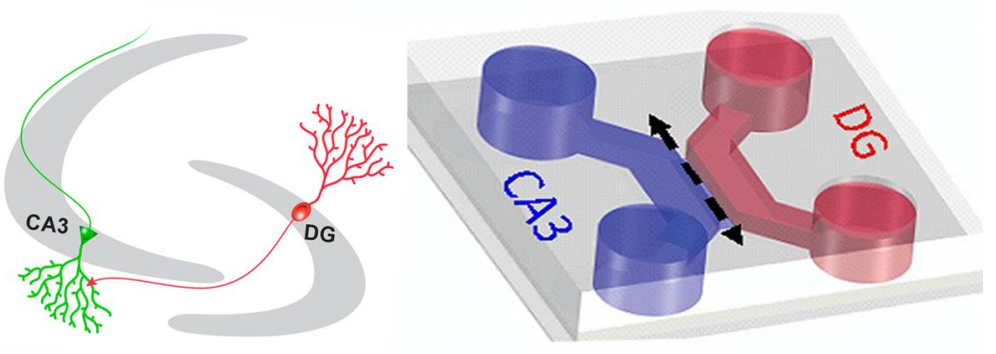

This assay is a way to study hippocampal connectivity. Dentate gyrus (DG) neurons and CA3 neurons can connect through openings in a tiny barrier. (Adapted with permission from ref. ${ }^{3}$, Elsevier)

a known factor in $\mathrm{AD}$ but hard to show in vitro, says system co-developer and UNC researcher Hansang Cho. While at MGH, he designed a chip to isolate microglia and activate them via soluble cues.

MGH researcher Doo Yeon Kim approached Cho to explore the integration of microfluidic devices into his lab's 3D culture models. Rudy Tanzi, also at MGH, guided the project, and the multidisciplinary partnership with several others continued after Cho moved to UNC. Their system involves a chamber with patient-derived neural stem cells differentiated into neurons and astrocytes. A microfluidic channel connects this chamber to one seeded with microglia. Several days after seeding, imaging showed microglia migrating toward the neurons and astrocytes and killing them.

Spires-Jones likes how the system architecture lets experimenters add microglia separately and only after neurons and astrocytes are established. "This cleverly gets around some tricky issues of growing all three cell types together at the same time and allows observation of migration of microglia into the area with the other cells," she says. She believes other labs will likely try the triculture system. "Microfluidics expertise may be a hurdle but these types of devices are getting easier to make, order, or even print in 3D," she says.

Neural progenitor cells offer many possibilities; they can be differentiated into neurons or astrocytes, and possibly oligodendrocytes, says Kim. In mixed neural culture, microglia are easily activated and become destructive. That behavior is typically kept in check with drugs, which the team sought to avoid.

The system shows an important difference between 2D and 3D cultures: genetically engineered $\mathrm{AD}$ neurons cultured in 2D did not show plaques or tangles, whereas in the 3D assay the team saw accumulating and aggregating pathogenic $\beta$-amyloid peptide, says Kim. The researchers have long wanted to model neuroinflammation. As Tanzi says, so-called resilient brains show abundant plaques and tangles but no cognitive decline and are usually free of neuroinflammation. "So we believe that plaque and tangles set the stage for neuroinflammation, which then destroys the bulk of the neurons, leading to dementia," he says.

The team is assessing how similar cell death in the model is to AD-related cell death. They are optimizing the system for high-throughput drug screening so it might be used to explore drug targets involved in neuronal death as well as amyloid and tau accumulation.

Overall, 3D culturing systems are better for modeling complex multicellular systems and recapitulating cell-cell interactions, says Kim, such as the interactions in a neural network or neural-glial interactions as in the triculture system. Several labs are finding that $3 \mathrm{D}$ conditions can accelerate neuronal differentiation and maturation more readily than $2 \mathrm{D}$. Yet $2 \mathrm{D}$ culture models offer a more homogeneous environment and a way to better synchronize cells for imaging. Experimental goals will determine the $2 \mathrm{D} / 3 \mathrm{D}$ choice, he says.

Thibault Honegger, a neuro-engineer at Neuro Engineering Technologies Research Institute (NETRI), a research institute with public and private funding that also incubates startups, likes how the triculture microfluidic system addresses a distinct scientific question, offers a macroscale view of the interaction between cell types and shows the stages of plaque and tau accumulation in AD. He is hopeful about modeling diseases of the brain in this fashion and about the prospects for the field of microfluidic neuro-engineering.

A former researcher at Centre National de la Recherche Scientifique in Grenoble, Honegger co-founded NETRI to build and validate microfluidic network models in neuroscience. The idea is to use microfluidics to build brain models that 'translate' connectome-level changes such as those associated with disease. He co-developed a device with reservoirs of neuronal populations plated in $2 \mathrm{D}$ culture that are then connected in a 3D configuration. Neurites expand into collagen-filled channels equipped with planar electrodes. Electrokinetic forces accelerate or slow down neurite expansion.

Since publishing their first microfluidic device in $2013^{5}$, Honegger and colleagues have been expanding their model.

"We actually connect millions of 'neurons' together and we try to reveal part of the brain circuitry," he says. To date they have linked up to 2.5 million neurons on a chip and the cells in these systems have survived for up to six months. A microfluidic environment is minimalistic and gives researchers much spatial and temporal control, he says. One can, for example, model the cortex by depositing 1 to 4 million neurons at one spot and connecting them in a physiologically relevant manner to other chambers.

In this system, neurons are not differentiated; the team extracts rat embryonic neurons from brain regions of interest such as hippocampus or cortex. A microfluidic neural network certainly lacks the complexity of an in vivo rat brain but, says Honegger, it offers in vitro experimental and control options not possible with organoids. In cell culture, the flow associated with changing media suffices to set neural stem cells on an unintended differentiation course. Unwanted neuronal cell types might proliferate and lead to too much heterogeneity in a dish. Such derailment can be avoided in a microfluidic chamber with flow control, he says.

In the early stages of $\mathrm{AD}$ or Parkinson's disease (PD), says Honegger, part of a circuit starts to degenerate in undetectable ways. In $\mathrm{PD}$, dopaminergic neurons begin dying but the neural network reconfigures itself to balance out this damage. That leads to functional recovery in spite of beginning structural damage. It's as if the brain were deciding, says Honegger, "Oh, here, it's not working well so we are going to process this information through other nodes." That's one aspect he hopes to model in a microfluidic network. He would also like

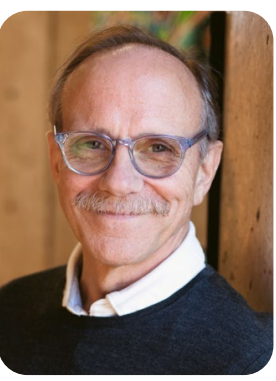

Increasing bonds between cell biology and bioengineering mean that $2 \mathrm{D}$ assays can be built into various 3D architectures, says Fred 'Rusty' Gage.

(Credit: Salk Institute) 
to study how a disease like PD spreads. Honegger sees a bright future for what he calls " $2.5 \mathrm{D}$ cultures," which implies a 3D projection onto a $2 \mathrm{D}$ assay.

\section{Organoids}

Some assays involve 3D clumps of cells such as spheroids that can be manipulated or screened. Other 3D assays tap into the ability of stem cells to self-assemble into organoids. The first such structure from the lab of Hans Clevers at Hubrecht Institute and University Medical Center in Utrecht involved using stem cells from intestinal crypts in mice to create crypt-villus-like structures in a dish. In terms of appearance, the authors note, "they were indistinguishable from those derived from whole crypts." Even without being embedded in a typical physiological niche in vivo, these self-organizing structures can "generate a continuously expanding, self-organizing epithelial structure of normal gut."

Much research has ensued related to organoids given the degree to which they can have the appearance, physiology and molecular traits of organs. At the Institute of Molecular Biotechnology in Vienna, Madeline Lancaster, Jürgen Knoblich and colleagues developed cerebral organoids ${ }^{6}$. Knoblich and his team recently created neoplastic cerebral organoids (neoCORs): gene-edited organoids for modeling brain cancer development in vitro ${ }^{7}$. These organoids carry mutations that are known initiators of brain cancer. The authors note that these neoCORs complement the current basic and preclinical models for studying brain tumor biology and could also be used for scaled-up targeted drug testing.

The scientists used transposon-mediated gene insertion and CRISPR-Cas9-based mutagenesis and found overlap in gene expression signatures between neoCORs and tumors. They generated two types of organoids: one with similarities to tumors that afflict children and young adults, so-called central nervous system primitive neuroectodermal tumors; and organoids that expressed genes typical of glioblastoma tumors. Labs have previously used spheroids as well as $2 \mathrm{D}$ assays to model brain tumors, and the neoCOR developers believe their organoids can "to a certain degree" mimic the in vivo structure of brain tumors. Their mix of healthy and cancer cells offers a view of the interactions between the two.

Organoid labs modeling the brain face considerable challenges. They can use two-photon imaging to structurally characterize organoids, says Honegger, but the technique is too slow to resolve circuitlevel changes such as those accompanying disease. When 3D culture models depend
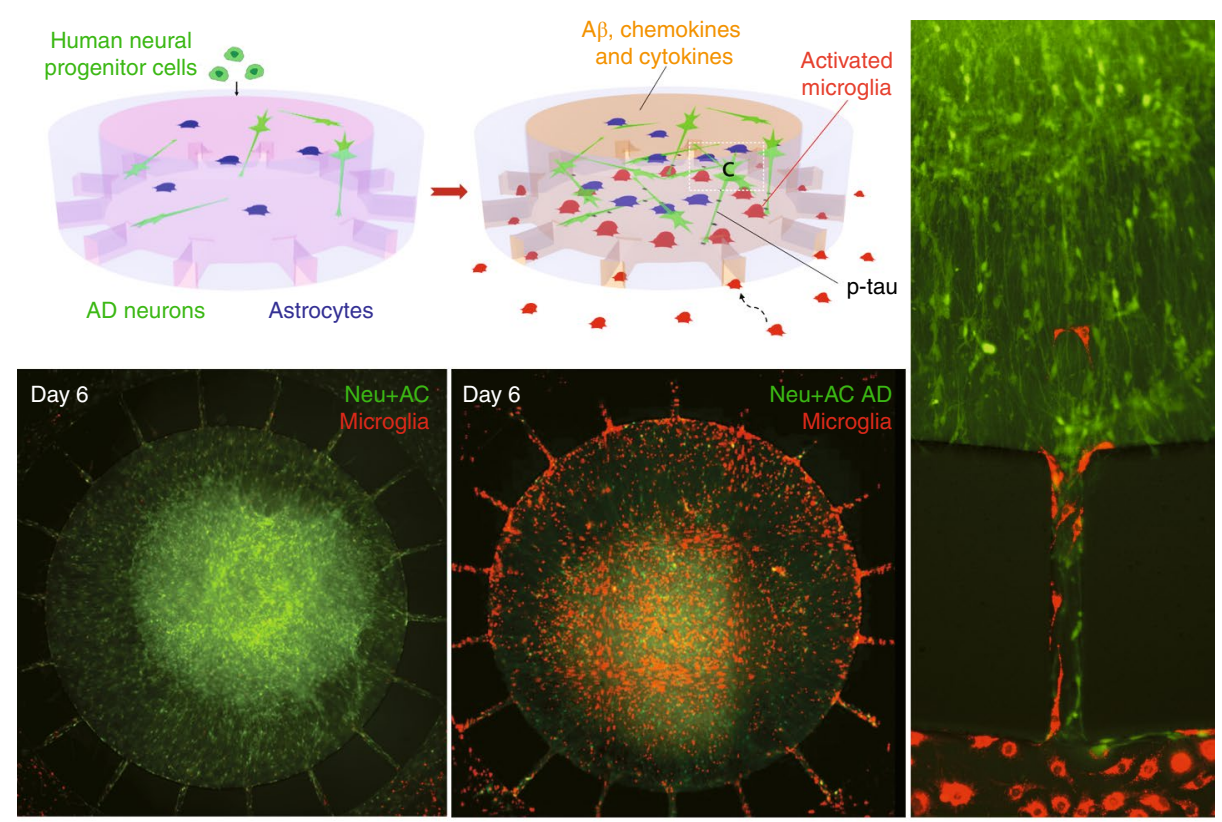

In this system, scientists mimic Alzheimer's disease (AD). Microglia migrate toward and attack neurons (Neu) and astrocytes (AC). (Adapted with permission from ref. ${ }^{4}$, Springer Nature)

on complicated self-organization processes, as is true with cerebral organoids, says Kim, "it is still technically challenging to achieve homogeneous and consistent cultures." Methods advances are needed to control local microenvironments to allow homogeneous organoid systems to emerge. With simpler 3D models such as the triculture system, homogeneous cultures are achieved more easily, he says, which positions a method for scale-up, such as for drug screening. With 3D culture models that use hydrogel and human neural stem cells, cell density can be controlled and adjusted as needed, just as in 2D cultures. "But again, you cannot control cell density for 3D models that depend on the self-organization process," he says.

\section{Hooked-up systems}

With organoids, an important drawback is their lack of vasculature. Once they are larger than around three millimeters in diameter, they begin to necrose in their core, says Gage. As this cell death occurs, the outermost cells can appear healthy but, he says, experimenters worry how changes in the core might affect their experiment.

Thinking about this led Gage and colleagues to graft organoids into highly vascularized locations in the brains of mice ${ }^{8}$. Once integrated into the vascular bed, "we were surprised, it was very efficient, we started seeing blood vessels," he says. They used two-photon microscopy to image around one millimeter in depth and saw that the organoids were thriving. There was essentially no cell death at nearly 250 days, when the team ended the experiment. Before grafting, they grew the organoids in vitro until around 45 days of age and a primitive neuroepithelium, a cortex-like structure, began to develop. Lancaster, now at Medical Research Council Laboratory of Molecular Biology, sees many advantages to this approach. There are certainly scalability issues, she says, but having the brain organoid in the context of a whole organism has important advantages for studying the interaction with other cells and tissues, such as microglia, or for studying gut-brain axis questions. Kim is also happy to see this

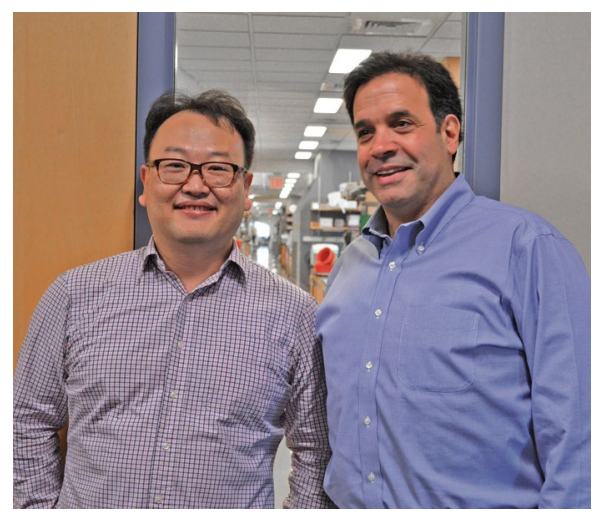

MGH researchers Doo Yeon Kim (left) and Rudy Tanzi (right) and UNC colleagues built a triculture model of Alzheimer's disease. Credit: MGH 
model and that a human organoid can be maintained and connected to the mouse brain's network and vasculature.

Gage and his team are now applying this model system to model autism, depression and bipolar disorder. These conditions are intertwined: some families appear to show genetic susceptibility. The scientists are also using the system to follow the emergence of the blood-brain barrier, using an injected dextran bead in the forming blood vessels. "We can look for the first time at the formation of human blood vessels during early development in an in vivo setting," he says. "So there's good biology there."

Developing this method took Gage back to his early career at the University of Lund, where he and his colleagues applied intracerebral grafting in rats to study neuronal maintenance. At the time, it was unknown whether nerve growth factor was active in the brain. "There weren't really good assays," he says. He set out to graft excised superior cervical ganglia. One graft was exposed to the hippocampal surface. For another graft, the transport of nerve growth factor from the hippocampus to the graft was cut off. Neural cell death occurred in locales without trophic support, while many neurons survived where exposed to nerve growth factorevidence for "neuronal survival factors" that maintain intracerebral connections. At the time the authors noted, "The intracerebral neural implantation technique should provide a valuable tool as an in vivo assay system for the further exploration of cerebral neuronotrophic mechanisms and their changes after damage with age."

Decades later, as Gage followed work with organoids and other labs bioengineering synthetic vascular systems, he decided to try grafting surgery. It had been years since his last surgery of this kind and only mid-surgery did he remember that the older work had been in rats, not mice. "It was actually a little bit humorous to recognize the fact that not only has all this time elapsed but I'm doing it in an organism one-fifth the size," he says. He says his postdoctoral fellows have quickly learned the method, and visitors come to the lab to learn it. Given the large number of methods papers, organoids have become quite standard, he says, but transferring organoids to a suitable location in the brain and performing quality control takes effort. Organoids still have a way to go, he says. Existing pancreatic organoids are "beautiful" and liver organoids are coming along. "I think this is an entire field," he says.

Gage also sees much promise with microfluidics and has recruited a bioengineer with a materials science background to his lab to work with colleagues at the University of California, San Diego, for machining

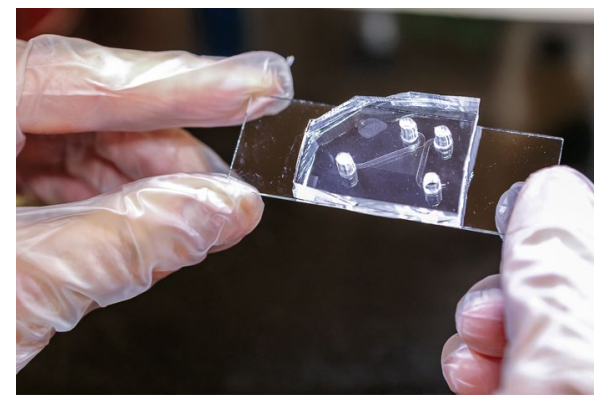

At NETRI, scientists are building brain models to 'translate' connectome-level changes. Credit: CNRS, NETRI

devices for brain modeling. Bioengineers are developing sophisticated artificial blood vessels and vascular systems to handle nutrient flow. Honegger has worked with a member of Harvard University researcher Jennifer Lewis's lab whose bioprinting approach generates fine synthetic vasculature. "It will be fantastic to make microfluidic blood vessel systems and microfluidic neural networks with our AD models," says Kim. For example, he says, one system with linked microfluidic chips models the blood vessel landscape and physiology of the blood-brain barrier. "In the end, our efforts to build 3D $\mathrm{AD}$ microfluidic models can be merged into these systems," says Kim.

Some cell types, such as microglia, are not represented in organoids, and this is an aspect Gage and others are working on. One could, says Gage, make microglia derived from patient cells and study, for example, what happens once they are grafted into a disease-free context. One could look at how responsible microglia are for the synaptic pruning that accompanies many neurodegenerative disorders. In a few studies, labs are integrating microglia into organoids, "but what I am more concerned about is whether they are really bona fide microglia," says Lancaster. Making microglia in vitro is proving difficult, she says, but directed differentiation protocols are improving.

Researchers are discussing what role the innate immune system might play in conditions such as Parkinson's disease (PD), says Gage. Posthumous analysis of tissue from PD patients has shown that $\mathrm{CD}^{+}$ T lymphocytes migrate to the brain. "We had the smoking gun with the pathology but we didn't have the experiment," he says. In a collaboration with researchers at Friedrich-Alexander-Universität Erlangen Nürnberg, the team co-cultured $\mathrm{CD}^{+}$cells from patients with dopamine neurons and found that the $\mathrm{CD} 4^{+}$cells produce IL-17, a cytokine toxic to neurons.

\section{How real is it?}

Models with 3D assays or organoids are producing AD pathology, says Tanzi. They are similar to aging brains, but they are not actual models of all aspects of brain aging. Andrew Yoo at Washington University, a former Tanzi lab member, is developing ways to generate human neurons that age. Modeling aged-brain conditions for studies of neurodegenerative diseases is one of the greatest challenges for 3D human brain models, says Kim. He and others have found that human iPSC-derived neurons express tiny amounts of four-repeat adult tau isoform, a protein needed to recapitulate neurofibrillary tangles. It's a starting point for a model in the making.

$3 \mathrm{D}$ cell culture models can create more realistic brain-like structures than 2D cultures with neurons and glial cells, but caution is advised before calling such systems 'real' biology, says Kim. Both model classes capture stages of development or disease and can, in many cases, replace in vivo models. 3D cultures can keep molecules within a matrix, says Spires-Jones, giving them a more "brainlike" local microenvironment. These molecules are diluted out or can be lost with media changes in $2 \mathrm{D}$ systems. "In contrast, monolayer ' $2 \mathrm{D}$ ' cultures have the advantage of being easier and I think still more reproducible experiment to experiment," she says.

In general, says Lancaster, $2 \mathrm{D}$ seems to be a better choice for high-throughput applications such as drug screening because of how similar the neighboring wells on a plate are. But 3D is better for modeling tissue architecture, she says. For disorders with morphological features, 3D would be expected to be a better model. "Thus, 3D is probably better suited for disease modeling and target identification in the first place, and then 2D may give you a system to develop drugs to those identified targets," she says.

\section{Vivien Marx \\ Technology editor for Nature Methods. \\ e-mail:v.marx@us.nature.com}

Published online: 30 October 2018 https://doi.org/10.1038/s41592-018-0191-z

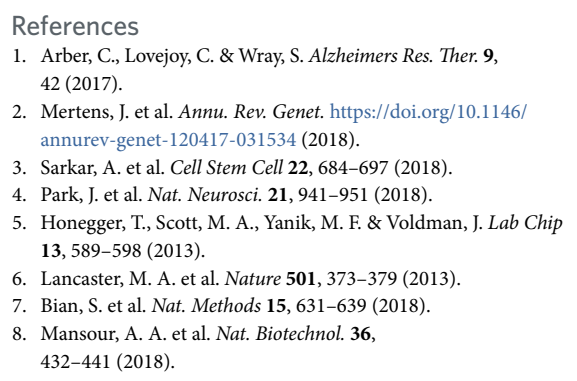

